\title{
Coupling of nanoparticle plasmons with molecular linkers
}

\author{
Nerea Zabala, ${ }^{a, b}$ Olalla Pérez-González, ${ }^{a, b}$ Peter Nordlander ${ }^{c}$ and Javier Aizpurua ${ }^{b}$ \\ ${ }^{a}$ Department of Electricity and Electronics, University of the Basque Country UPV/EHU, \\ Bilbao, Spain. \\ ${ }^{b}$ Donostia International Physics Center (DIPC) and Centro de Física de Materiales (CFM), \\ Centro Mixto CSIC-UPV/EHU, Donostia-San Sebastián, Spain. \\ ${ }^{c}$ Laboratory for Nanophotonics, Rice University, Houston, USA.
}

\begin{abstract}
In this work we study how plasmon modes of gold dimers are affected by a molecular bridge connecting both particles. Different models for the linker are considered to envisage the relation between the spectral changes observed in the extinction spectra and the electronic transport through the molecules. Depending on the size and nature of the molecular linker two different modes, known as BDP (Bonding Dimer Plasmon) and CTP (Charge Transfer Plasmon), are excited. Furthermore, when the molecular linker has an excitonic resonance, new spectral features emerge due to the plasmon-exciton coupling.
\end{abstract}

Keywords: Plasmon, nanoparticle, dimer, conductance, exciton, plexciton

\section{INTRODUCTION}

In the last decade there has been a great activity in the field of nanophotonics, aimed to understand and design devices capable of controlling light at the nanoscale. ${ }^{1-4}$ In particular nanoshells and metallic nanoparticles have emerged as fundamental structures due to the tunability of their plasmonic resonances and ability to enhance electromagnetic fields, which make them good candidates for different applications in biosensing or cancer research. ${ }^{5,6}$

When two metallic nanoparticles are placed close to each other, forming a dimer, new plasmonic modes are formed, which are interpreted as the hybridization of the plasmonic resonances of the individual nanoparticles. ${ }^{7-10}$ For non-touching dimers the optical response is mainly governed by the Bonding Dimer Plasmon (BDP) resonance, arising from the coupling of the dipolar modes of the individual particles. This mode presents strong charge densities of opposite sign at both sides of the inter-particle cavity, producing enormously enhanced local electromagnetic fields. For touching particles ${ }^{11}$ or, when a thin conductive path is opened between the nanoparticles, ${ }^{12}$ a new Charge Transfer Plasmon (CTP) mode is excited, in which the whole dimer acts as a dipolar plasmon mode, so that in the oscillations both particles present net charges of opposite sign.

At the same time electronic transport through molecules has developped as a main field in nanoscience, due to its potential technological applications in electronic devices. ${ }^{13-17}$ Recently, there has been a growing interest to relate plasmonic modes and molecules, intended to develop active plasmonic components for light harvesting, switches or modulators. ${ }^{18-20}$ Following the emerging interest in connecting molecular electronics and plasmonics, we studied the optical properties of plasmonic cavities formed by gold nanoshell dimers connected by a conductive bridge characterized by a simple model of pure conductor. ${ }^{12}$ This system can be considered as a first approach to describe a dimer connected by an ensemble of molecules. We identified two regimes depending on the conductance of the linker. In the low conductance regime, the optical response depends on the conductivity of the junction and the BDP mode blue-shifts into a Screened Bonding Dimer Plasmon SBDP. In the large conductance regime, a CTP mode appears red-shifted with respect to the SBDP. Using simple time scale arguments, we stablished the conductance thresholds for the appearance of these modes.

Further author information: (Send correspondence to N. Z.)

N.Z.: E-mail: nerea.zabala@ehu.es, Telephone: +34 946012538

O.P.G.: E-mail: olalla_perez@ehu.es, Telephone: +34 946015371

P.N.:E-mail:nordland@rice.edu, Telephone:(713)348-5171

Plasmonics: Metallic Nanostructures and Their Optical Properties IX, edited by Mark I. Stockman,

Proc. of SPIE Vol. 8096, 80961L · C 2011 SPIE · CCC code: 0277-786X/11/\$18 · doi: 10.1117/12.893776

Proc. of SPIE Vol. 8096 80961L-1 


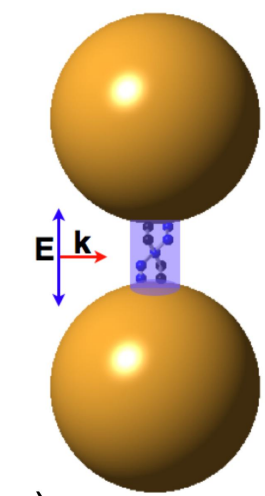

a)
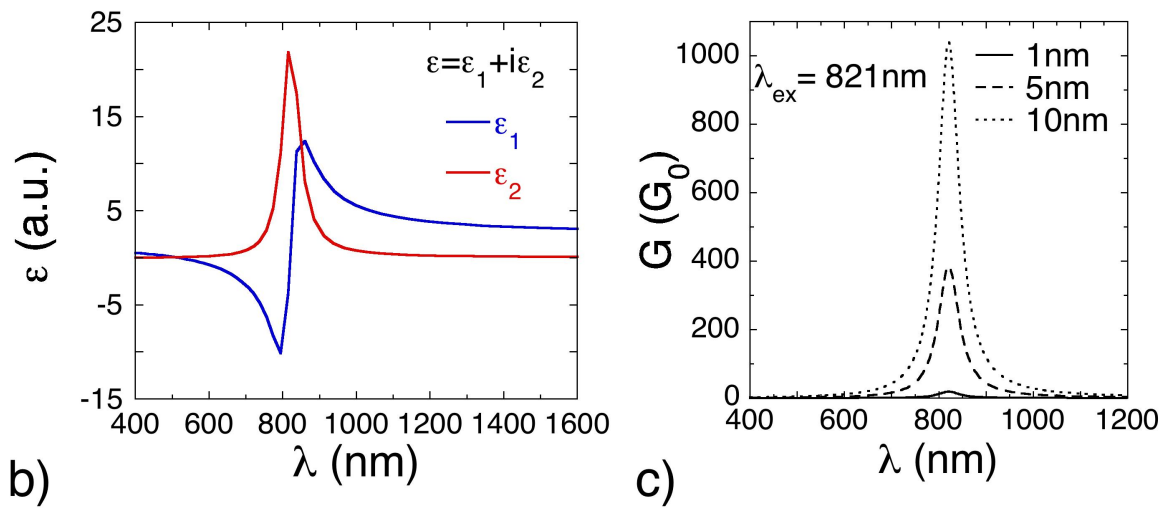

Figure 1. a) Two gold nanoparticles of radius $R=50 \mathrm{~nm}$ connected by a molecular linker mimicked by a solid cylinder of radius $a$ and length $d=1 \mathrm{~nm}$, corresponding to the minimum distance between the particles. b) Real and imaginary parts of the Drude-Lorentz dielectric function used to mimic the molecular bridge, for $\lambda_{e x}=821 \mathrm{~nm}$ (rotaxane) and reduced oscillator strenght $f=1.5$ and $\gamma=0.1 \mathrm{eV}$. c) Conductance for linkers of three different widths, as a function of the wavelength.

In this paper we extend our previous study by considering a simple Drude-Lorentz dielectric function to account for the excitonic transitions of the molecules connecting the dimer cavity. Excitonic transitions couple to the plasmon modes of the cavity giving rise to plasmon-exciton states, ${ }^{18,21-24}$ also called plexcitons. These mixed states are attractive due to their applications for SERS and optical devices. We study the emergence of the plexcitons for different sizes and characteristic resonant frequencies of the molecular linker. We believe that the understanding of the spectral changes in plasmonic nanocavities bridged by molecules may help to probe electrical transport at visible frequencies, a regime not accessible through electrical measurements.

\section{MODEL}

We have performed calculations for two identical gold particles of radius $R=50 \mathrm{~nm}$, characterized by an experimental local dielectric function $\epsilon(\omega)$ taken from the literature, ${ }^{25}$ with a gap $d=1 \mathrm{~nm}$ between them. The molecular linker is modeled as a cylinder of radius $a$ and length $d$, so that the caps of the cylinder fit perfectly to the spherical surfaces of the particles, as shown in Fig. 1a). Its dielectric response is modeled with a Drude-Lorentz model:

$$
\varepsilon(\omega)=1-\frac{f \omega_{e x}^{2}}{\left(\omega^{2}-\omega_{e x}^{2}\right)+i \omega \gamma},
$$

where $\omega_{e x}$ is the natural oscillator frequency of the molecular transition, $\gamma$ is the damping and $f$ describes the reduced oscillator strength. The real and imaginary parts of this function are represented in Fig. 1b) for rotaxane molecules ${ }^{21}$ having a unique transition resonance at energy $\hbar \omega_{e x}=1.51 \mathrm{eV}$, corresponding to $\lambda_{e x}=821 \mathrm{~nm}$. We have considered $\gamma=0.1 \mathrm{eV}$ anf $f=1.5$.

As in a previous work, in which we modeled the linker as a pure metal, we are interested in studying how the different cavity modes are tuned as the conductance of the linker is increased. In this model the conductance is determined by the geometry of the linker, which takes into account the spherical endings of the linker, and its conductivity $\sigma(\omega)=\sigma_{1}(\omega)+i \sigma_{2}(\omega)$, which in turn is related to the dielectric function as $\varepsilon(\omega)=1+i \frac{4 \pi \sigma(\omega)}{\omega}$ :

$$
G(\omega)=\sigma_{1}(\omega) \pi\left\{\sqrt{R^{2}-a^{2}}-R+(d / 2+R) \ln \left[1+2\left(R-\sqrt{R^{2}-a^{2}} / d\right]\right\} .\right.
$$

Note that for thin linkers, the conductance is close to $G=\pi a^{2} \sigma_{1}(\omega) / d$. We express the conductance in terms of the number $n$ of conductance quanta $G_{0}=2 e^{2} / h \simeq 77.5 \mu S$, as in molecular electronics, i.e., $G=n G_{0}$. Within the present model the dynamical conductance $G(\omega)$ is a lorentzian function centered at the excitonic resonance wavelength, whose intensity depends on the thickness and the reduced oscillator strength of the linker and its width depends on the damping. It is represented in Fig. 1c) for three different radii of the linker $a=1,5$ and 

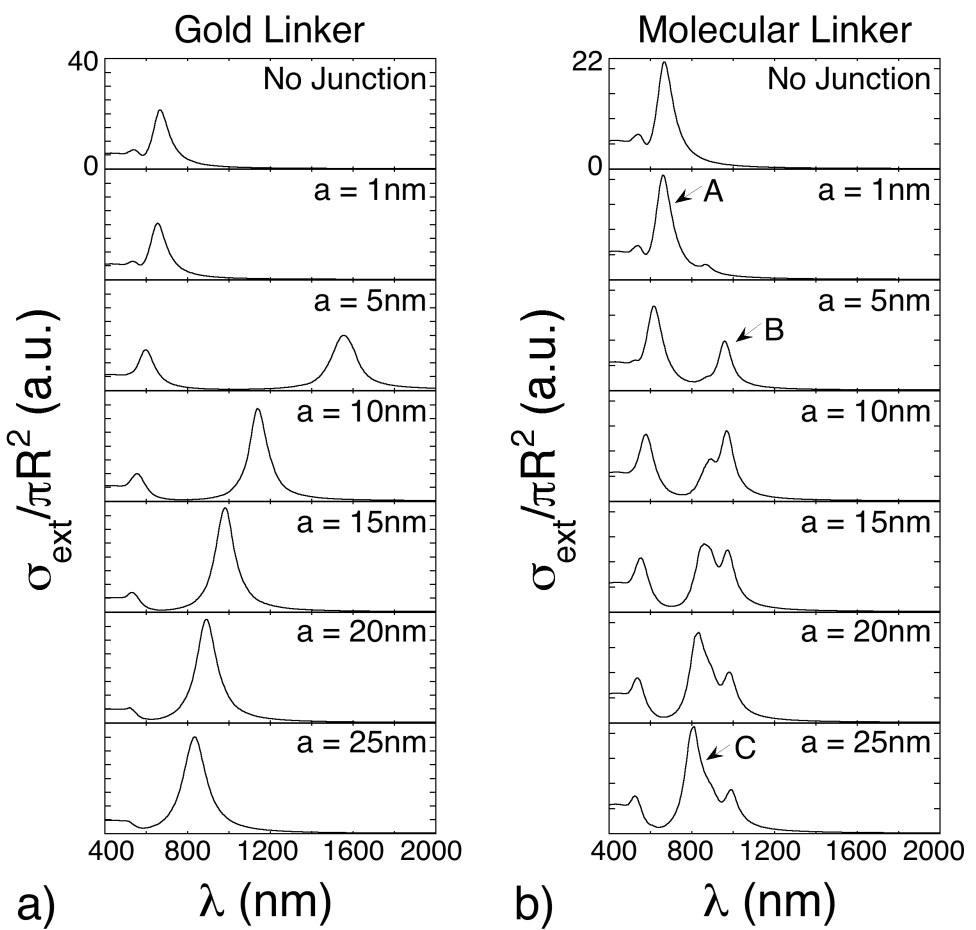

Figure 2. Extinction spectra of two gold nanoparticles of radius $50 \mathrm{~nm}$ connected by a) a gold linker and b) a molecular linker constituted by rotaxane molecules, as the radius $a$ of the junction is increased from 1 to $25 \mathrm{~nm}$. The extinction spectrum corresponding to the disconnected dimer is also plotted for comparison in both cases.

$10 \mathrm{~nm}$. To study the optical properties of the linked cavities in nanoparticle dimers we calculate the optical extinction spectra when polarized light with the electric field along the linker is incident from the left (see Fig. 1a)). We solve Maxwell's equations using the Boundary Element Method (BEM) ${ }^{26,27}$ with the geometrical variables and dielectric functions described above as inputs, to obtain the electromagnetic fields everywhere in the system.

\section{RESULTS}

Now we focus on the extinction spectra of linked gold spherical nanoparticle dimers. First, it is crucial to check the spectrum for the disconnected dimer. For the radius and distance considered in the present work $(R=50 \mathrm{~nm}$, $d=1 \mathrm{~nm}$ ), the corresponding extinction spectrum is displayed on top of Figs. 2a) and 2b). The main peak around $\lambda=670 \mathrm{~nm}$ is the Bonding Dimer Plasmon (BDP) excitation in the cavity, which corresponds to the hybridization of the dipolar modes $(l=1)$ of the particles, so that charges of opposite sign are concentrated at both sides of the interparticle cavity, and the field enhancement in this region is very large. The weak peak around $\lambda=540 \mathrm{~nm}$ corresponds to the hybridization of the quadrupolar modes $(l=2)$ of the individual particles.

The optical properties of dimers connected by pure metals were studied in a previous work, ${ }^{12}$ where we analyzed the effect of the conductance of the linker. We observed that as the conductance is increased by increasing the radius of the linker, or the conductivity of the medium, the BDP mode is blue-shifted and, for a critical value of the conductance a new mode emerges at higher wavelengths, which corresponds to the hybridization of two monopolar modes $(l=0)$ in the spheres and is not allowed for isolated nanoparticles. This is the so called CTP mode, because it corresponds to net charge flow along the linker as the plasmonic oscillations of the dimer take place. This mode was discussed previously by Romero et al. ${ }^{11}$ for touching gold dimers.

As a reference, to compare with molecular linkers, we show in Fig. 2a) the spectra of gold dimers connected by cylindrical gold junctions of different radii $a=1-25 \mathrm{~nm}$. As the radius is increased, we observe a blue shift of the BDP peak or screened mode SBDP, corresponding to a decrease of the capacitance in the cavity, as we discussed in reference 12. This mode hybridizes with the quadrupolar mode. But what is more important, a new 


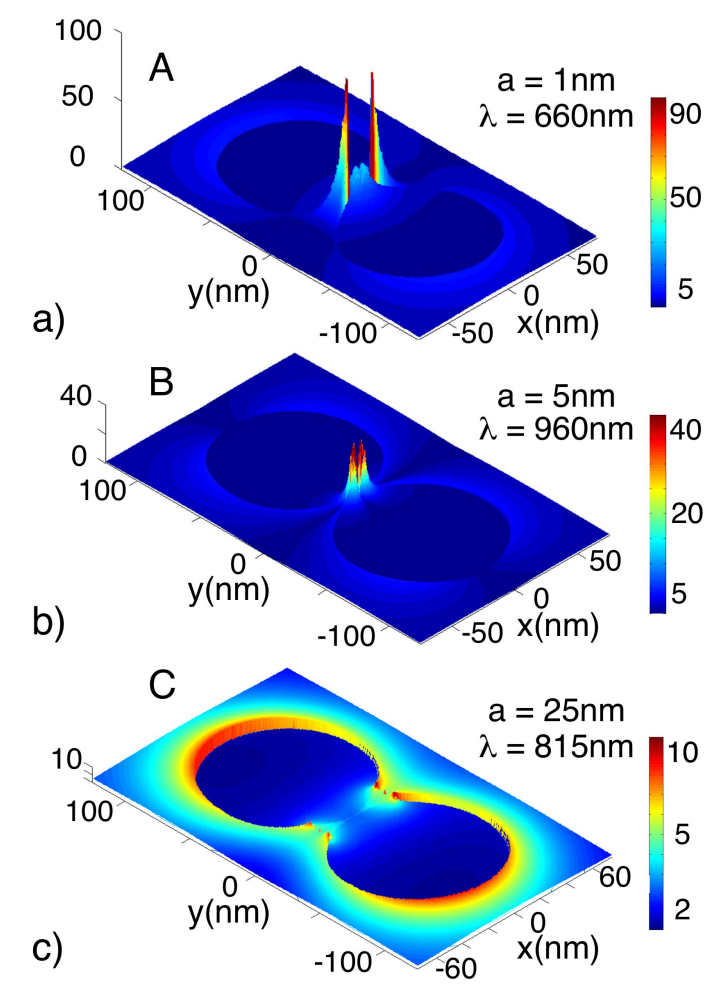

Figure 3. Electric field intensity $|E|$ maps in the transversal cross section of a gold dimer cavity connected by molecular linkers of different widths at the wavelengths corresponding to the peaks marked $\mathrm{A}, \mathrm{B}$ and $\mathrm{C}$ in Fig. 2b). Incident light comes from the negative side of the $x$ axis, with the electric field polarized along the $y$ axis.

mode of CTP character emerges for the gold linker of radius $a=5 \mathrm{~nm}$ at $\lambda=1555 \mathrm{~nm}$. This system corresponds to a conductance in the linker $G=702 G_{0}$, which is above the threshold for the emergence of this mode, estimated in $G_{C T P}=468 G_{0}$ from a simple argument, ${ }^{12}$ which takes into account the relation between the period of the plasmon oscillations in the cavity and the current through the junction. As the radius of the linker is increased up to $25 \mathrm{~nm}$, a dramatic blue shift, increase of intensity and further saturation are observed. As we discussed, ${ }^{12}$ the emergence of this mode is accompained by a reduction of the field enhancement in the cavity and specially in the central part of the linker, corresponding to a current flow mainly along the walls.

Let us analyze now the spectra obtained for rotaxane molecular linkers $\left(\lambda_{e x}=821 \mathrm{~nm}\right)$ displayed in Fig. $\left.2 \mathrm{~b}\right)$. Note that in this case we use a different scale in the vertical axis, as it is clear from the dimer without linker at both sides on top of the figure. For the BDP of the cavity we observe a similar behaviour to the gold linker, but instead of the single CTP mode a double peak structure appears. For the thinest linker, corresponding to $a=1 \mathrm{~nm}$, the main peak (peak A) is slightly blue-shifted from the position of the disconnected dimer, around 660 $\mathrm{nm}$, and a shoulder appears around $865 \mathrm{~nm}$. For thicker junctions $(a>10 \mathrm{~nm})$, there is double peak structure around the wavelength of the excitonic resonance of the molecular linker considered. The peak of the shorter wavelength, around $\lambda=815 \mathrm{~nm}$, which we label as $\mathrm{C}$, gains strength as the linker is wider, so that at $a=25 \mathrm{~nm}$ it is the main excitation. The peak at longer wavelength, which we label as $\mathrm{B}$, behaves in the opposite way, i.e., loosing weight for wider junctions. This behaviour is due to the coupling of the exciton with the plasmonic modes of the cavity, giving rise to mixed states called plexcitons, ${ }^{18,22,24}$ as we will detail below.

In order to gain insight into the nature of these states, we plot in Fig. 3 three selected maps of the intensity of the electric field in linked dimers at the wavelengths corresponding to peaks marked as A, B and C in Fig. $2 \mathrm{~b}$ ), corresponding to $\lambda=660,960$ and $815 \mathrm{~nm}$ and radius $a=1,5$ and $25 \mathrm{~nm}$, respectively. The structure of the maps corresponding to peaks A and B resembles the BDP pattern, with the hot spot in the center of the cavity, but there are differences between them and with respect to the BDP patter of the dimer connected by 

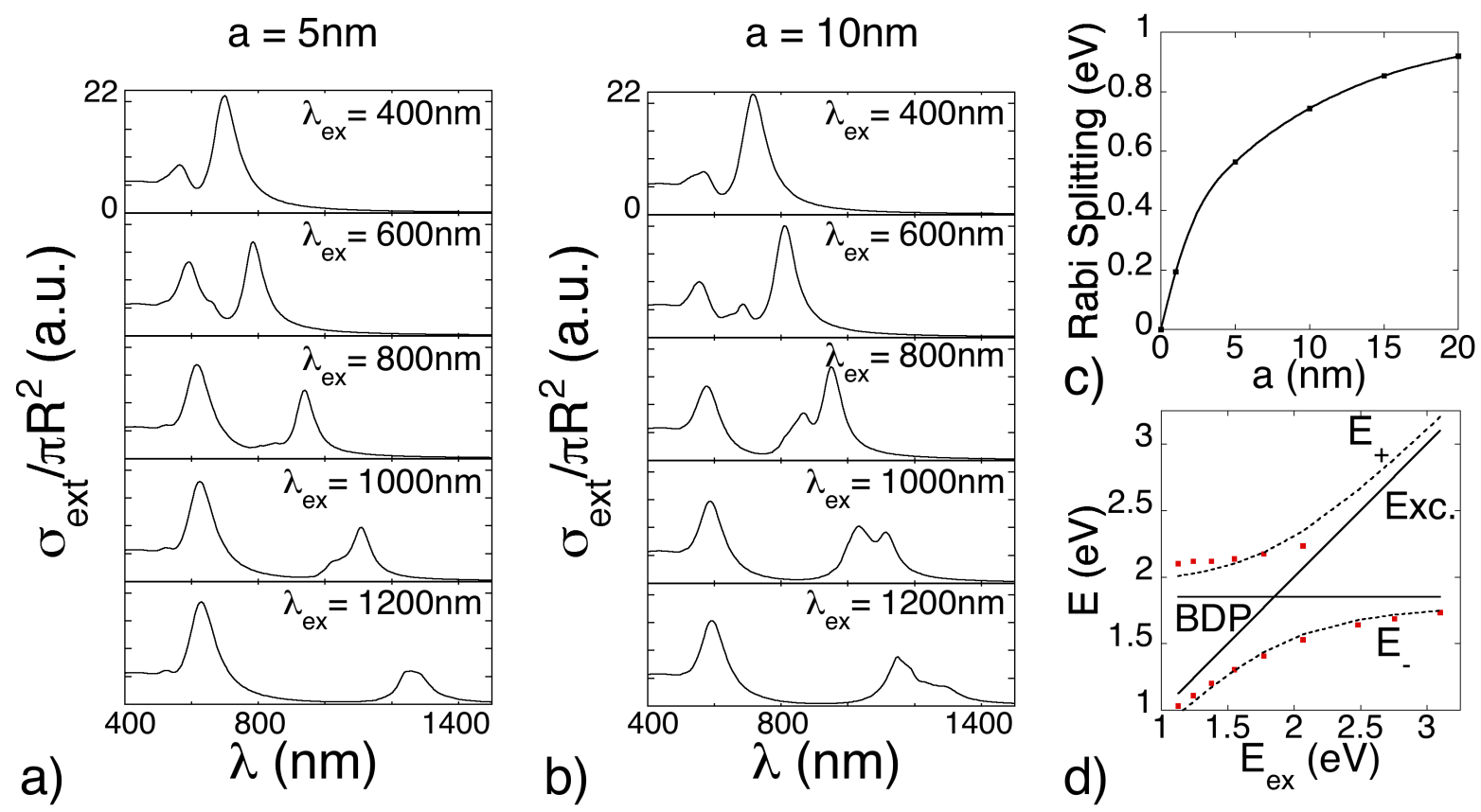

Figure 4. Extinction spectra of two gold nanoparticles of radius $50 \mathrm{~nm}$ connected by molecular linkers of radius a) $a=5 \mathrm{~nm}$ and b) $a=10 \mathrm{~nm}$ and different excitonic resonances $\lambda_{e x}=400-1200 \mathrm{~nm}$. c) Rabi splitting of the BDP mode as a function of the radius. d) $E_{+}$and $E_{-}$plexciton branches showing the anticrossing behaviour around the BDP and exciton lines.

a metal as for example gold, as in Fig. 2a). In both cases there is a decrease of intensity towards the axis of the linker but the field distribution is different. For peak A it is more concentrated in the outer part of the cavity, but for peak B it is more focused in the center. Furthermore, the dipolar distributions of the individual particles are oriented in a different way, for peak $\mathrm{A}$ it looks more like in the conventional BDP, but for peak $\mathrm{B}$ the individual dipoles are oriented in the transverse direction (perpendicular to the axis of the linker). Peak $\mathrm{C}$, on the other side, corresponds to the CTP pattern. So, we conclude that for the molecular linker the CTP moves to shorter wavelengths, so that it is between two mixed exciton-BDP modes. Note that for the linker with $a=10 \mathrm{~nm}$ the conductance is close to $G=1000 G_{0}$, while for $a=5 \mathrm{~nm}$ it is below $G=400 G_{0}$ (see Fig. 1c)), so that the conductance threshold estimated from the time argument used in reference 12 also holds for the molecular linkers, if we take into account the maximum value of the dinamical conductance $G(\omega)$. In fact, for the geometry considered, the threshold value is $G_{C T P}=\omega_{C T P} R^{2} / 4 \pi \sim 685 G_{0}$.

The splitting of the BDP mode of the cavity may be described with a simple model of two coupled oscillators corresponding to the exciton of the linker and the dipolar BDP plasmon cavity mode. ${ }^{28,29}$ Ignoring damping effects, the allowed energy eigenvalues of the coupled system are then given by:

$$
E_{ \pm}=\frac{E_{B D P}+E_{e x}}{2} \pm\left[\left(\frac{\hbar \Omega_{R}}{2}\right)^{2}+\frac{1}{4}\left(E_{B D P}-E_{e x}\right)^{2}\right]^{1 / 2}
$$

where $E_{B D P}$ and $E_{e x}$ are the energies of the BDP mode and the exciton respectively, and $\hbar \Omega_{R}$ is the Rabi splitting. The magnitude of the Rabi splitting is obtained from our calculations when $E_{B D P}=E_{e x}$. This coupling then gives rise to two energy branches, $E_{ \pm}$, which correspond to the peaks A and B in Fig. 2. The peak of lower energy, $E_{-}$, longer $\lambda$, is peak B and that of higher energy $E_{+}$corresponds to peak A.

The corresponding dispersion curves of the new modes present an anticrossing behaviour around the lines of the exciton and the BDP mode (see Fig. 4d)). This anticrossing effect can be observed in Figure 4, where optical spectra for two linkers of radius $a=5 \mathrm{~nm}$ and $a=10 \mathrm{~nm}$ are shown for different wavelenghts of the excitonic transition. For $\lambda_{e x}=400 \mathrm{~nm}$ there is no apparent coupling, the spectrum resembles that of the disconnected dimer. But as $\lambda_{e x}$ increases, we observe a slight grow and shift of the peak around $\lambda=600 \mathrm{~nm}$ (corresponding to $\left.E_{+}\right)$and a further saturation for higher wavelengths. The peak corresponding to $E_{-}$shifts more with $\lambda_{e x}$, 
but showing a progressive decrease in intensity. This behaviour is similar in Figs 4a) and b), but for $a=10 \mathrm{~nm}$ another peak appears in between, starting at $\lambda_{e x}=600 \mathrm{~nm}$ and then also shifts and gains weight with respect to the $E_{-}$peak.

Note that in the two oscillator model we have taken into account only the BDP dipolar mode. This explains that in Fig. 4d) the agreement of the points extracted from the spectra with the $E_{+}$branch is not so good, due to the hybridization with the quadrupolar mode. The Rabi splitting, giving the distance between both plexciton peaks when $\lambda_{e x}=\lambda_{B D P}$, depends on the radius and, therefore, on the conductance of the junction, as displayed in Fig. 4c). Its value is of several hundreds of meVs, but there is an increase and further saturation as the radius is increased.

\section{CONCLUSIONS}

We have discussed the effects of molecular linkers in the optical spectra of gold nanoparticle dimers, using a simple Drude-Lorentz to account for the excitonic transitions of the molecules. We have observed the appearence of new features in addition to the BDP and CTP modes studied previously for pure metal bridges. On one side, the mixing of the BDP mode with the exciton gives rise to a splitting into two mixed modes called plexcitons, which we have characterized by the Rabi splitting, given by a simple model of two coupled oscillators. On the other side, the CTP mode is blue-shifted with respect to the metallic connection.

\section{ACKNOWLEDGMENTS}

This project was supported by the Etortek project inanoGUNE from the Basque Government (BG), project FIS2010-19609-C02-01 from the Spanish Ministry of Science and Innovation, grant IT-366-07 from BG-UPV/EHU and project CUBIHOLE EUI2008-03816 from European Union. Computational resources were provided by IZOSGIker (UPV/EHU, MICINN, BG, ESF).

\section{REFERENCES}

[1] L. Novotny and B. Hecht, Principles of Nano-Optics, Cambridge University Press, Cambrdge, England, 2006.

[2] S. Maier, Plasmonics: Fundamentals and Applications, Springer, New York, 2007.

[3] M. Pelton, J. Aizpurua, and G. W. Bryant, "Metal-nanoparticle plasmonics," Laser 63 Photon. Rev. 2, pp. 136-159, 2008.

[4] S. Lal, S. Link, and N. J. Halas, "Plasmonics: nanoscale optics from sensing to waveguiding," Nature Photonics 1, pp. 641-648, 2008.

[5] A. McFarland and R. V. Duyne, "Single silver nanoparticles as real-time optical sensors with zeptomole sensitivity," Nano Lett. 3, pp. 1057-1062, 2003.

[6] N. J. Halas, S. Lal, W. Chang, S. Link, and P. Nordlander, "Plasmons in strongly coupled metallic nanostructures," Chem. Reviews 111, pp. 3913-3961, 2011.

[7] P. Nordlander, C. Oubre, E. Prodan, K. Li, and M. I. Stockman, "Plasmon hybridization in nanoparticle dimers," Nano Lett. 4, pp. 899-903, 2004.

[8] D. Kim, J. Heo, S. Ahn, S. Han, W. Yun, and Z. Kim, "Real-space mapping of the strongly coupled plasmons of nanoparticle dimers," Nano Lett. 9, pp. 3619-3625, 2009.

[9] D. Kim, J. Heo, S. Ahn, S. Han, W. Yun, and Z. Kim, "Plasmon hybridization in individual gold nanocrystal dimers: Direct observation of bright and dark modes," Nano Lett. 10, pp. 632-637, 2010.

[10] J. Zuloaga, E. Prodan, and P. Nordlander, "Quantum description of the plasmon resonances of a nanoparticle dimer," Nano Lett. 9, pp. 887-891, 2009.

[11] I. Romero, J. Aizpurua, F. J. G. de Abajo, and G. W. Bryant, "Plasmons in nearly touching metallic nanoparticles: singular response in the limit of touching dimers," Opt. Express 14, pp. 9988-9999, 2006.

[12] O. Pérez-González, N. Zabala, A. Borisov, N. Halas, P. Nordlander, and J. Aizpurua, "Optical spectroscopy of conductive junctions in plasmonic cavities," Nano Lett. 10, pp. 3090-3095, 2010.

[13] H. Park, J. Park, A. Lim, E. Anderson, A. Alivisatos, and P. McEuen, "Nano-mechanical oscillations in a single-c60 transistor," Nature 407, pp. 57-60, 2000. 
[14] G. V. Nazin, X. H. Qiu, and W. Ho, "Visualization and spectroscopy of a metal-molecule-metal bridge," Science 302, pp. 77-81, 2003.

[15] J. Liao, J. Agustsson, S. Wu, C. Schonenberger, M. Calame, Y. Leroux, M. Mayor, O. Jeannin, Y.-F. Ran, S.-X. Liu, and S. Decurtins, "Cyclic conductance switching in networks of redox-active molecular junctions," Nano Lett. 10, pp. 759-764, 2010.

[16] L. Venkataraman, J. Klare, I. Tam, C. Nuckolls, M. Hybertsen, and M. Steigerwald, "Single-molecule circuits with well-defined molecular conductance," Nano Lett. 6, pp. 458-462, 2006.

[17] P. Banerjee, D. Conklin, S. Nanayakkara, T.-H. Park, M. J. Therien, , and D. A. Bonnell, "Plasmon-induced electrical conduction in molecular devices.," ACS Nano 4, pp. 1019-1025, 2010.

[18] G. Wurtz, P. Evans, W. Hendren, R. Atkinson, W. Dyckson, R. Pollard, and A. Zayats, "Molecular plasmonics with tunable exciton-plasmon coupling strength in j-aggregate hybridized au nanorod assemblies," Nano Lett. 7, pp. 1297-1303, 2007.

[19] E. Hendry, F. García-Vidal, L. Martín-Moreno, J. Gómez-Rivas, M. Bonn, A. Hibbins, and M. Lockyear, "Optical control over surface-plasmon-polariton-assisted thz transmission through a slit aperture," Phys. Rev. Lett. 100, pp. 123901-123904, 2008.

[20] P. Andrew and W. Barnes, "Energy transfer across a metal film mediated by surface plasmon polaritons," Science 306, pp. 1002-1005, 2004.

[21] Y. Zheng, Y. Yang, L. Jensen, L. Fang, B. Juluri, A. Flood, P. Weiss, J. Stoddart, and T. Huang, "Active molecular plasmonics: controlling plasmon resonances with molecular switches," Nano Lett. 9, pp. 819-825, 2009.

[22] N. Fofang, T. Park, O. Neumann, N. Mirin, P. Nordlander, and N. Halas, "Plexcitonic nanoparticles: Plasmon-exciton coupling in nanoshell-j-aggregate complexes," Nano Lett. 8, pp. 3481-3487, 2008.

[23] D. Gómez, K. Venon, P.Mulvaney, and T. Davis, "Surface plasmon mediated strong exciton-photon coupling in semiconductor nanocrystals," Nano Lett. 10, pp. 274-278, 2010.

[24] A. Manjavacas, F. G. de Abajo, and P. Nordlander, "Quantum plexcitons: strongly interacting plasmons and excitons," Nano Lett. 11, pp. 2318-2323, 2011.

[25] P. Johnson and R. Christy, "Optical constants of the noble metals," Phys. Rev. B 6, pp. 4370-4379, 1972.

[26] F. G. de Abajo and A. Howie, "Relativistic electron energy loss and electron-induced photon emission in inhomogeneous dielectrics," Phys.Rev. Lett. 80, pp. 5180-5183, 1998.

[27] F. G. de Abajo and A. Howie, "Retarded field calculation of electron energy loss in inhomogeneous dielectrics," Phys. Rev. B 65, pp. 115418-17, 2002.

[28] S. Rudin and T. Reinecke, "Oscillator model for vacuum rabi splitting in microcavities," Phys. Rev. B 59, pp. 10227-10233, 1999.

[29] L. Andreani, G. Panzarini, and J. Gérard, "Strong-coupling regime for quantum boxes in pillar microcavities: Theory," Phys. Rev. B 60, pp. 13276-13279, 1999. 